\title{
3D Reconstruction of Metallic Surfaces by Photopolarimetric Analysis
}

\author{
P. d'Angelo and C. Wöhler \\ DaimlerChrysler Research and Technology, Machine Perception \\ P. O. Box 2360, D-89013 Ulm, Germany \\ \{pablo.d_angelo, christian.woehler\}@daimlerchrysler.com
}

\begin{abstract}
In this paper we present a novel image-based 3D surface reconstruction technique that incorporates both reflectance and polarisation features into a variational framework. Our technique is especially suited for the difficult task of $3 \mathrm{D}$ reconstruction of rough metallic surfaces. An error functional consisting of several error terms related to the measured reflectance and polarisation properties is minimised in order to obtain a $3 \mathrm{D}$ reconstruction of the surface. We show that the combined approach strongly increases the accuracy of the surface reconstruction result, compared to techniques based on either reflectance or polarisation alone. We evaluate the algorithm based on synthetic ground truth data. Furthermore, we report 3D reconstruction results for a raw forged iron surface, thus showing the applicability of our method in real-world scenarios, here in the domain of quality inspection in the automotive industry.
\end{abstract}

\section{Introduction}

Three-dimensional surface reconstruction has become an important technique in the context of industrial quality inspection. The currently most widely used active approaches are primarily based on the projection of structured light (for a survery, cf. e. g. [1]). While such methods are accurate, they require a highly precise mutual calibration of cameras and structured light sources. Multiple structured light sources may be needed for 3D reconstruction of non-convex surfaces. Hence, for inline quality inspection of industrial part surfaces, less intricate passive image-based techniques are desirable.

A well-known passive image-based surface reconstruction method is shape from shading. This approach aims at deriving the orientation of the surface at each pixel by using a model of the reflectance properties of the surface and knowledge about the illumination conditions - for a detailed survey, cf. e. g. 22. The integration of shadow information into the shape from shading formalism is described in detail in $[8$.

A further approach to reveal the 3D shape of a surface is to make use of polarisation data. For smooth dielectric surfaces, the direction and degree of polarisation as a function of surface orientation are governed by elementary physical 
laws as described in detail e. g. in [5]. Information about the polarisation state of light reflected from the surface is utilised in [5] to reconstruct the 3D shape of transparent objects, involving multiple light sources equally distributed over a hemisphere and a large number of images acquired through a linear polarisation filter at different orientations. Reflectance and polarisation properties of metallic surfaces are examined in [9], but no physically motivated polarisation model is derived. In 7 polarisation information is used to determine the orientation of a surface. Applications of such shape from polarisation approaches to real-world scenarios, however, are rarely described in the literature.

In this paper we present an image-based method for 3D surface reconstruction based on the simultaneous evaluation of information about reflectance and polarisation. The corresponding properties of the surface material are obtained by means of a series of images acquired through a linear polarisation filter under different orientations. Both reflectance and polarisation features are integrated into a unified variational framework. The method is systematically evaluated based on a synthetically generated surface to obtain information about its accuracy and is applied to the real-world example of a raw forged iron surface.

\section{Combination of Reflectance and Polarisation Features for 3D Surface Reconstruction}

In our scenario, we will assume that the surface $z(x, y)$ to be reconstructed is illuminated by a point light source and viewed by a camera, both situated at infinite distance in the directions $\boldsymbol{s}$ and $\boldsymbol{v}$, respectively. Parallel incident light and an orthographic projection model can thus be assumed. For each pixel location $(u, v)$ of the image we intend to derive a depth value $z(u, v)$. The surface normal is given in gradient space by the vector $\boldsymbol{n}=(-p,-q, 1)^{T}$ with $p=\partial z / \partial x$ and $q=\partial z / \partial y$. The incidence angle $\theta_{i}$ is defined as the angle between $\boldsymbol{n}$ and $\boldsymbol{s}$, the emission angle $\theta_{e}$ as the angle between $\boldsymbol{n}$ and $\boldsymbol{v}$, and the phase angle $\alpha$ as the angle between $\boldsymbol{s}$ and $\boldsymbol{v}$. The observed pixel intensity is denoted by $I(u, v)$, the corresponding modelled intensity by the reflectance function $R(\boldsymbol{n}, \boldsymbol{s}, \boldsymbol{v})$. The $3 \mathrm{D}$ surface reconstruction formalism we utilise throughout this paper is related to the shape from shading scheme described in detail in [2, 3, 4]. It relies on the global minimisation of an error function that consists of a weighted sum of several individual error terms. One part of this error function is the intensity error term

$$
e_{I}=\sum_{u, v}[I(u, v)-R(\boldsymbol{n}, \boldsymbol{s}, \boldsymbol{v})]^{2} .
$$

A regularization constraint $e_{s}$ is introduced which requires local continuity of the surface. A smooth surface implies small absolute values of the directional derivatives of the surface gradients. In this paper we will therefore make use of the error term (cf. also [2, 4])

$$
e_{s}=\sum_{u, v}\left[p_{x}^{2}+p_{y}^{2}+q_{x}^{2}+q_{y}^{2}\right] .
$$


Alternatively, one might replace (2) by the departure from integrability error term described in detail in 3 .

In this paper we regard metallic surfaces with a strongly non-Lambertian reflectance function $R(\boldsymbol{n}, \boldsymbol{s}, \boldsymbol{v})$. We can assume, however, that the surface interacts with the incident light in an isotropic manner in the sense that the reflectance function exclusively depends on $\theta_{i}, \theta_{e}$, and $\alpha$. It will be determined by means of a suitable measurement procedure (cf. Section 3).

In our scenario, the incident light is unpolarised. For smooth metallic surfaces the light remains unpolarised after reflection at the surface. Rough metallic surfaces, however, cause the reflected light to be partially polarised $[9$. When observed through a linear polarisation filter, the reflected light will have a transmitted radiance that oscillates sinusoidally as a function of the orientation of the polarisation filter between a maximum $I_{\max }$ and a minimum $I_{\min }$. The polarisation angle $\Phi \in\left[0^{\circ}, 180^{\circ}\right]$ denotes the orientation under which the maximum transmitted radiance $I_{\max }$ is observed. The polarisation degree is defined by $D=\left(I_{\max }-I_{\min }\right) /\left(I_{\max }+I_{\min }\right) \in[0,1]$. As no physical model exists so far which is able to accurately describe the polarisation properties of rough metallic surfaces, the functions $R_{\Phi}(\boldsymbol{n}, \boldsymbol{s}, \boldsymbol{v})$ and $R_{D}(\boldsymbol{n}, \boldsymbol{s}, \boldsymbol{v})$ describing polarisation angle and degree of the material, respectively, have to be determined empirically (cf. Section 31).

To integrate polarisation features into the 3D surface reconstruction framework, we define the error terms

$$
e_{\Phi}=\sum_{u, v}\left[\Phi(u, v)-R_{\Phi}(\boldsymbol{n}, \boldsymbol{s}, \boldsymbol{v})\right]^{2}
$$

and

$$
e_{D}=\sum_{u, v}\left[D(u, v)-R_{D}(\boldsymbol{n}, \boldsymbol{s}, \boldsymbol{v})\right]^{2}
$$

which denote the mean square deviation between observed and modelled polarisation angle and degree, respectively. To obtain surface gradients $p(u, v)$ and $q(u, v)$ that optimally fit the observed reflectance and polarisation properties, the overall error

$$
e=e_{s}+\lambda e_{I}+\mu e_{\Phi}+\nu e_{D}
$$

has to be minimzed. The Lagrange parameters $\lambda$, $\mu$, and $\nu$ denote the relative weights of the error terms. We obtain an iterative update rule for $p(u, v)$ and $q(u, v)$ by setting the derivatives of $e$ with respect to $p(u, v)$ and $q(u, v)$ to zero (see also [4):

$$
p_{n+1}=\bar{p}_{n}+\lambda(I-R) \frac{\partial R}{\partial p}+\mu\left(\Phi-R_{\Phi}\right) \frac{\partial R_{\Phi}}{\partial p}+\nu\left(D-R_{D}\right) \frac{\partial R_{D}}{\partial p}
$$

with $\bar{p}_{n}$ as a local average. An analogous expression is obtained for $q$. The initial values $p_{0}(u, v)$ and $q_{0}(u, v)$ must be provided based on a-priori knowledge about the surface (cf. Section 4). Numerical integration of the gradient field, employing e. g. the algorithm described in [4, yields the surface profile $z(u, v)$. The partial derivatives are computed at $\left(\bar{p}_{n}, \bar{q}_{n}\right)$, respectively. 


\section{Measurement of Photopolarimetric Image Data}

This section explains how the reflectance and polarisation properties of the surface are measured and described in terms of suitable analytical functions for further processing.

\subsection{Measurement of Reflectance Properties}

The reflectance function of a typical rough metallic surface consists of three components: a diffuse (Lambertian) component, the specular lobe, and the specular spike 6. The diffuse component is generated by internal multiple scattering processes. The specular lobe, which is caused by single reflection at the surface, is distributed around the specular direction and may be rather broad. The specular spike is concentrated in a small region around the specular direction and represents mirror-like reflection, which is dominant in the case of smooth surfaces. For an illustration, see Fig. 17.
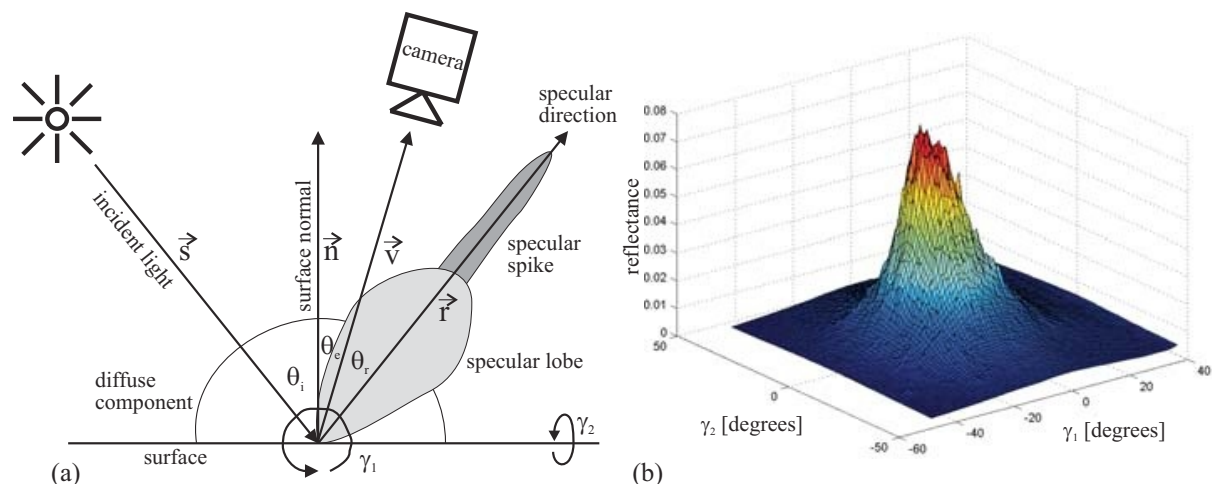

(b)

Fig. 1. (a) Plot of the three components of the reflectance function (cf. also [6]). (b) Measured reflectance of a raw forged iron surface. In the least mean squares fit of (7) to these measurements, the specular lobe is described by $\sigma_{1}=4.49$ and $m_{1}=6.0$, the specular spike by $\sigma_{2}=1.59$ and $m_{2}=57.3$

With $\theta_{i}$ as the incidence angle and $\theta_{r}$ as the angle between the specular direction $\boldsymbol{r}$ and the viewing direction $\boldsymbol{v}$ (cf. Fig. 1a), which can be expressed as $\cos \theta_{r}=$ $2 \cos \theta_{i} \cos \theta_{e}-\cos \alpha$, we define an analytical form for the reflectance function:

$$
R\left(\theta_{i}, \theta_{e}, \alpha\right)=\rho\left[\cos \theta_{i}+\sum_{n=1}^{N} \sigma_{n} \cdot\left(2 \cos \theta_{i} \cos \theta_{e}-\cos \alpha\right)^{m_{n}}\right] .
$$

For $\theta_{r}>90^{\circ}$, only the diffuse component is considered. The albedo $\rho$ is assumed to be constant over the surface. We set $N=2$ to model both the specular lobe and the specular spike. The coefficients $\left\{\sigma_{n}\right\}$ denote the strength of the specular components relative to the diffuse component, while the parameters $\left\{m_{n}\right\}$ denote their widths. 
To obtain reflectance measurements of a surface, a sample part is attached to a goniometer, which allows for a rotation of the sample around two orthogonal axes. The corresponding goniometer angles $\gamma_{1}$ and $\gamma_{2}$ can be adjusted at an accuracy of a few arcseconds. As illustrated in Fig. 17, adjusting $\gamma_{1}$ is equivalent to rotating the surface normal $\boldsymbol{n}$ around an axis perpendicular to the plane spanned by the vectors $\boldsymbol{s}$ and $\boldsymbol{r}$, while adjusting $\gamma_{2}$ causes a rotation of $\boldsymbol{n}$ around an axis lying in that plane. The phase angle $\alpha$, given by the relative position of the light source and the camera, is assumed to be constant over the image. It is straightforward to determine the surface normal $\boldsymbol{n}$, the incidence angle $\theta_{i}$, and the emission angle $\theta_{e}$ from the goniometer angles $\gamma_{1}$ and $\gamma_{2}$ and the vectors $\boldsymbol{s}$ and $\boldsymbol{v}$.

The average greyvalue over an image area containing a flat part of the sample surface is regarded as the reflectance (in arbitrary units) under the given illumination conditions, respectively. A typical reflectance measurement is shown in Fig. 11b.

\subsection{Measurement of Polarisation Properties}

The measurement of the polarisation properties of the surface is similar to the reflectance measurement. For each configuration of goniometer angles, five images are acquired through a linear polarisation filter at an orientation angle $\omega$ of $0^{\circ}, 45^{\circ}, 90^{\circ}, 135^{\circ}$, and $180^{\circ}$. For each filter orientation $\omega$, an average pixel intensity over an image area containing a flat part of the sample surface is computed as described in Section 3.1. A function of the form

$$
I(\omega)=I_{c}+I_{v} \cos (\omega-\Phi)
$$

is then fitted to the measured pixel intensities. The filter orientation $\Phi$ for which maximum intensity is observed corresponds to the polarisation angle defined in Section 2. The polarisation degree now becomes $D=I_{v} / I_{c}$. In principle, three measurements would be sufficient to determine the three parameters $I_{c}, I_{v}$, and $\Phi$, but the fit becomes less noise-sensitive when more measurements are used. The value $I_{c}$ represents the reflectance of the surface.

As no accurate physically motivated model for the polarisation properties of rough metallic surfaces is available so far, we perform a polynomial fit in terms of the goniometer angles $\gamma_{1}$ and $\gamma_{2}$ to the measured polarisation angle and degree. The polarisation angle is represented by an incomplete third-degree polynomial of the form

$$
R_{\Phi}\left(\gamma_{1}, \gamma_{2}\right)=a_{\Phi}+b_{\Phi} \gamma_{2}+c_{\Phi} \gamma_{1} \gamma_{2}+d_{\Phi} \gamma_{1}^{2} \gamma_{2}+e_{\Phi} \gamma_{2}^{3}
$$

which is antisymmetric in $\gamma_{2}$, and $R_{\Phi}\left(\gamma_{1}, 0\right)=a_{\Phi}=$ const, corresponding to coplanar vectors $\boldsymbol{n}, \boldsymbol{s}$, and $\boldsymbol{v}$. In an analogous manner, the polarisation degree is represented by an incomplete polynomial of the form

$$
R_{D}\left(\gamma_{1}, \gamma_{2}\right)=a_{D}+b_{D} \gamma_{1}+c_{D} \gamma_{1}^{2}+d_{D} \gamma_{2}^{2}+e_{D} \gamma_{1}^{2} \gamma_{2}^{2}
$$

which is symmetric in $\gamma_{2}$. The symmetry properties are required for geometrical reasons as long as an isotropic interaction between incident light and surface 

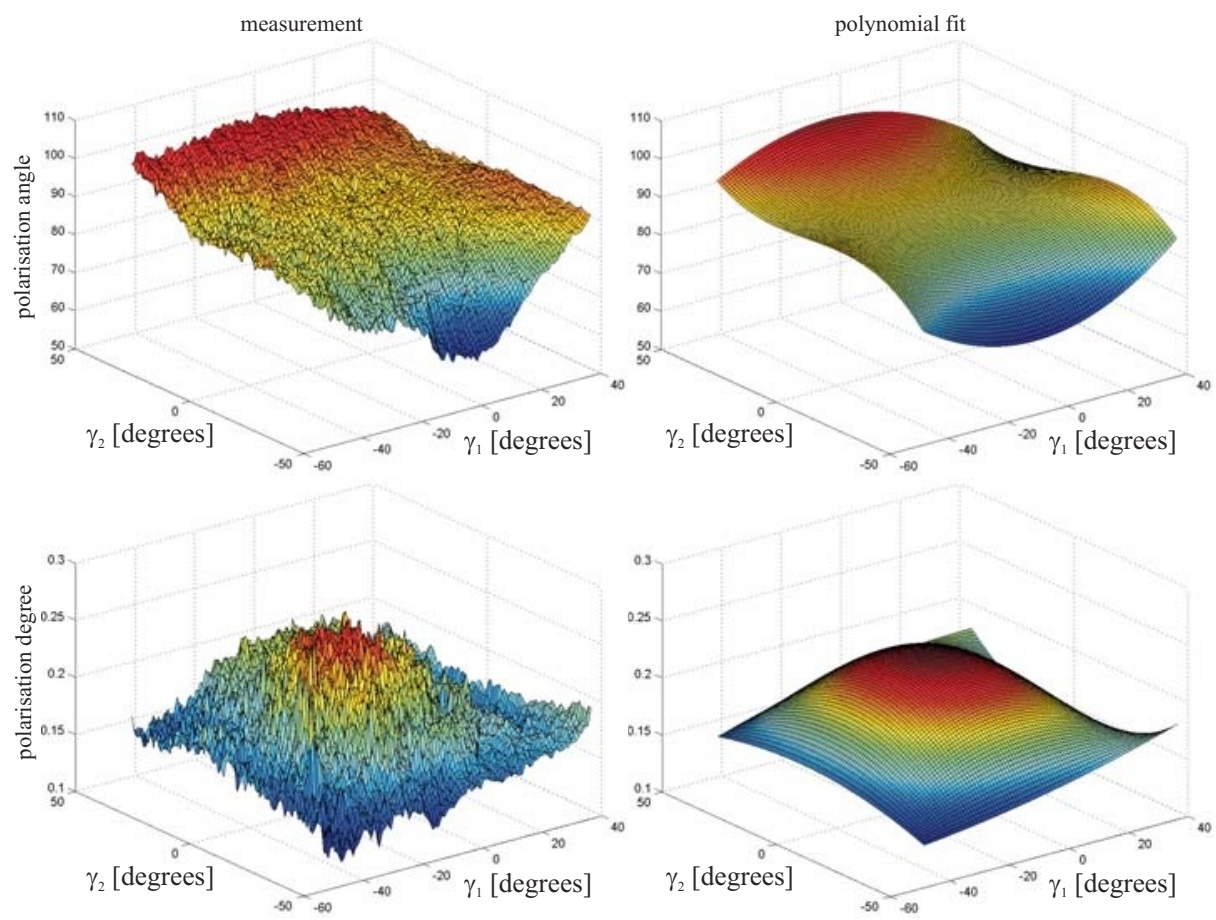

Fig. 2. Measured and modelled polarisation properties of a raw forged iron surface. Top: polarisation angle. Bottom: polarisation degree

material can be assumed. The polarisation properties of a raw forged iron surface measured at a phase angle of $\alpha=79^{\circ}$ are illustrated in Fig. 2, along with the polynomial fits according to (9) and (10).

\section{Experimental Results}

\subsection{Evaluation Based on Synthetic Ground Truth Data}

To examine the quality of $3 \mathrm{D}$ reconstruction, dependent on which reflectance and polarisation features are used, we apply the algorithm described in Section 2 to the synthetically generated surface shown in Fig. 3 a. We assume a perpendicular view on the surface along the $z$ axis. The scene is illuminated in image $u$ direction under an angle of $11^{\circ}$ with respect to the horizontal plane, resulting in a phase angle of $\alpha=79^{\circ}$. The surface albedo $\rho$ is computed based on the specular reflections, which appear as regions of maximum intensity $I_{\mathrm{spec}}$ and for which we have $\theta_{r}=0^{\circ}$ and $\theta_{i}=\theta_{e}=\alpha / 2$, such that from (7) we obtain

$$
\rho=I_{\text {spec }} \cdot\left[\cos (\alpha / 2)+\sum_{n=1}^{N} \sigma_{n}\right]^{-1}
$$




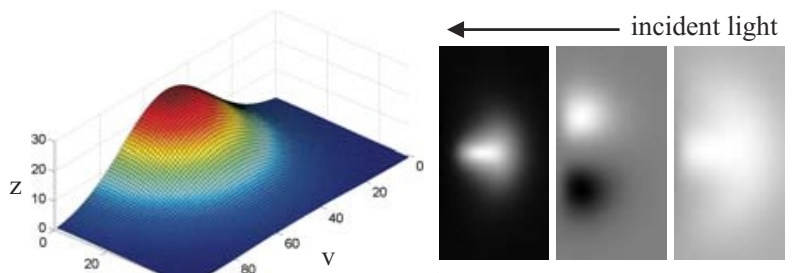

(a) ${ }^{20} \mathrm{u}{ }_{100}{ }^{80} \quad$ (b)
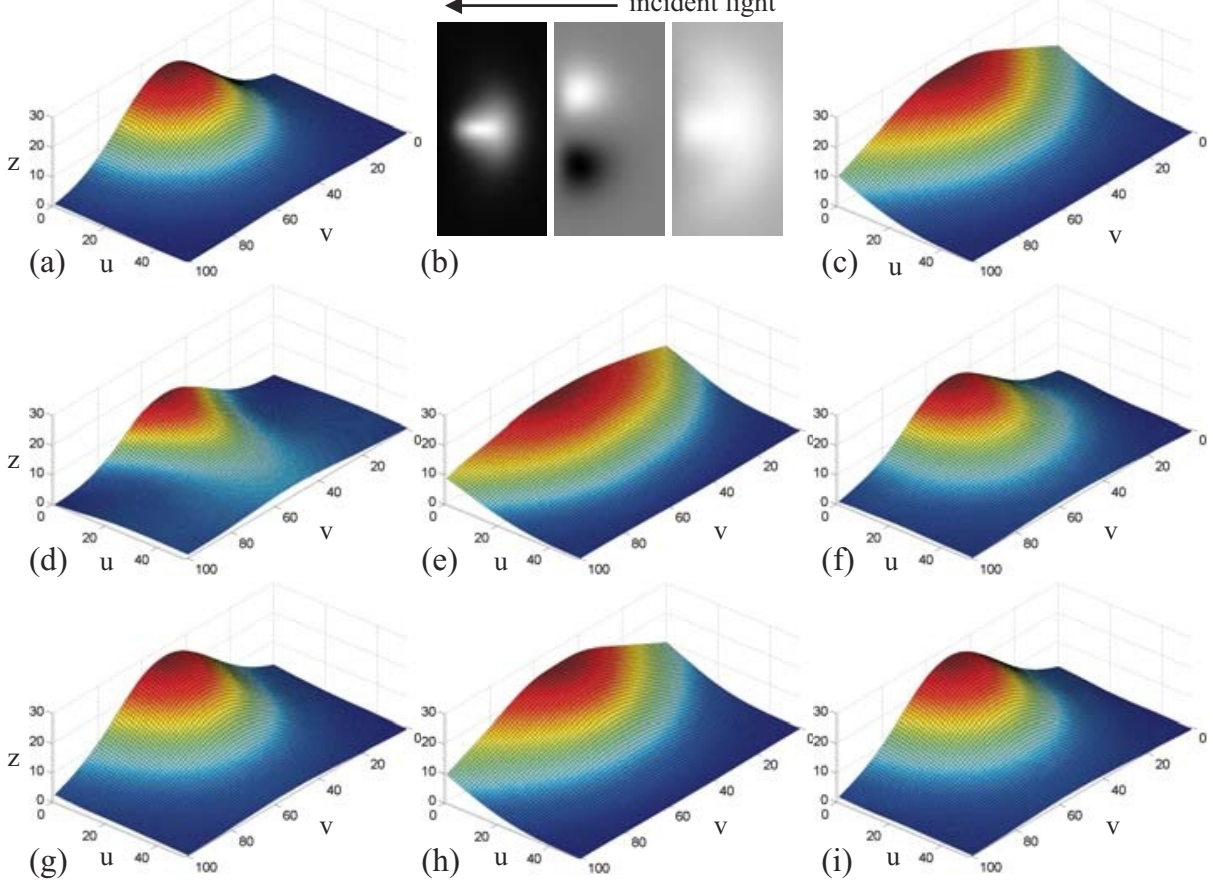

Fig. 3. 3D reconstruction of a synthetically generated surface. (a) Ground truth. (b) From the left: Reflectance image, polarisation angle image, polarisation degree image. The 3D reconstruction result is obtained based on (c) reflectance, (d) polarisation angle, (e) polarisation degree, (f) polarisation angle and degree, (g) reflectance and polarisation angle, $(\mathrm{h})$ reflectance and polarisation degree, (i) reflectance, polarisation angle and degree. The reconstruction results have been obtained based on noise-free images

The initial values for $p(u, v)$ and $q(u, v)$ must be provided relying on approximate knowledge about the surface orientation. In the synthetic surface example, $p(u, v)$ and $q(u, v)$ are initialised with zero values.

The reflectance, polarisation angle, and polarisation degree images shown in Fig. 3b have been generated by means of the polynomial fits to the measured reflectance and polarisation properties presented in Figs. 1 and 2, The weights for the corresponding error terms according to (5) are set to $\lambda=0.0075$ ( $I$ in arbitrary units, with a maximum of the order 0.1 ), $\mu=4.0$ ( $\Phi$ in radian), and $\nu=0.8$ ( $D$ between 0 and 1$)$. When an error term is neglected, the corresponding weight is set to zero. The $3 \mathrm{D}$ reconstruction results obtained based on all possible combinations of reflectance and polarisation features are shown in Fig. 3 c-i. The corresponding RMS deviations from the ground truth for $z, p$, and $q$ are given in Table 1. The best 3D reconstruction result is obtained when all three features are used, while the second-best result is achieved for a combination of reflectance and polarisation angle. According to Table 1, the performance of our method hardly decreases when noise is added to the images. 
Table 1. Results of evaluation on synthetic ground truth data. Noise level is \pm 0.5 for the reflectance (maximum value 6.74 ), $\pm 4^{\circ}$ for the polarisation angle and \pm 0.05 for the polarisation degree. The noise is uniformly distributed over the corresponding intervals. The residual error in (g) and (i) in the noise-free case is primarily due to the assumption of a smooth surface (2)

\begin{tabular}{lcccccccc}
\hline & & \multicolumn{3}{c}{ RMS error } & \multicolumn{4}{c}{ RMS error } \\
Features & Figure 3 & \multicolumn{2}{c}{ (without noise) } & \multicolumn{3}{c}{ (with noise) } \\
& & $z$ & $p$ & $q$ & $z$ & $p$ & $q$ \\
\hline Reflectance & (c) & 2.95 & 0.072 & 0.331 & 2.95 & 0.075 & 0.331 \\
Pol. angle & (d) & 4.24 & 0.177 & 0.088 & 4.06 & 0.165 & 0.097 \\
Pol. degree & (e) & 3.50 & 0.131 & 0.330 & 3.47 & 0.130 & 0.330 \\
Pol. angle and degree & (f) & 3.87 & 0.076 & 0.039 & 4.48 & 0.077 & 0.059 \\
Reflectance and pol. angle & (g) & 0.45 & 0.030 & 0.047 & 0.70 & 0.044 & 0.062 \\
Reflectance and pol. degree & (h) & 2.85 & 0.074 & 0.326 & 2.85 & 0.076 & 0.327 \\
Reflectance and polarisation & (i) & 0.37 & 0.017 & 0.034 & 0.69 & 0.035 & 0.056 \\
(angle and degree) & & & & & & & \\
\hline
\end{tabular}

Reflectance $R$ and polarisation degree $R_{D}$ appear to contain somewhat redundant information, as their combination hardly reduces the reconstruction error, compared to the results obtained by means of one the features alone. Both $R$ and $R_{D}$ display a maximum in the specular direction $\left(\theta_{r}=0^{\circ}\right)$ and decrease in a similar manner for $\theta_{r}>0^{\circ}$. The dependence on surface orientation, however, is much lower for $R_{D}$ than for $R$, while the measurement error tends to be significantly smaller for the reflectance. In practical applications (cf. Section 4.2) it turns out that 3D surface reconstruction should preferrably be performed based on a combination of reflectance and polarisation angle, neglecting the polarisation degree.

\subsection{Application to a Rough Metallic Surface}

In this section, we will describe the application of our 3D surface reconstruction method to the raw forged iron surface of an automotive part. For such a kind of surface, the polarisation degree for specular reflection tends to vary over the surface by up to 20 percent, depending on the locally variable microscopic roughness, in a rather erratic manner (cf. Fig. 4a). In contrast, the behaviour of the polarisation angle turns out to be very stable over the surface. The polarisation degree is thus an unreliable feature, such that we perform a $3 \mathrm{D}$ reconstruction of the forged iron surface based on a combination of reflectance and polarisation angle. For each pixel, polarisation angle and degree are determined as described in Section 3. The surface albedo is obtained according to (11). As the microscopic surface roughness produces a strong scatter in the measured polarisation data, the images are blurred before the polarisation properties are computed. This is permitted because we desire to obtain information about the surface shape on scales significantly larger than the microscopic roughness. 


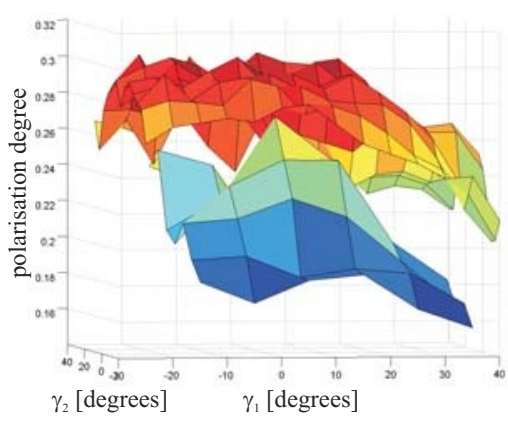

(a) (b)

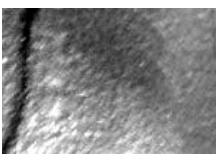

(c)

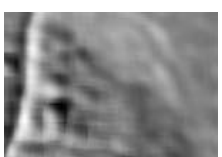

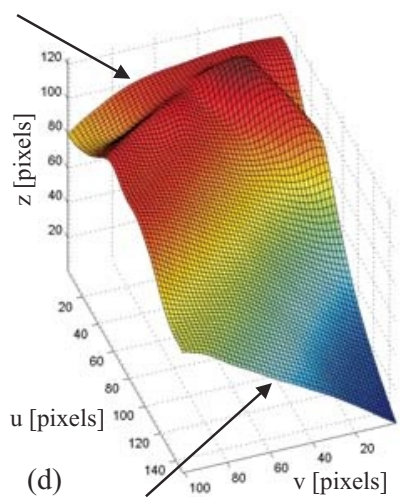

(d)

Fig. 4. Application of the described 3D surface reconstruction method to a raw forged iron surface. (a) Polarisation degree, measured at two different locations of the same industrial part consisting of forged iron. (b) Reflectance image, original (above) and blurred (below). The greyvalues are scaled logarithmically. (c) Polarisation angle image. (d) Reconstructed 3D profile. Due to a fault caused during the forging process, the cross-section of the surface significantly changes its shape over the image (arrows)

The $3 \mathrm{D}$ reconstruction along with the reflectance and polarisation images is shown in Fig. 4 b-d. At the left image border, the cross-section of the surface is roughly cylindrical, while it is flat at the right image border (arrows). The ground truth, i. e. independently derived accurate $z(u, v)$ quantities, is not available for this surface, but the real surface shows a very similar asymmetric behaviour. A flawless part would display the cylindrical cross-section (upper arrow) over the complete surface region shown in Fig. $4 \mathrm{~d}$. The observed asymmetric shape is due to a fault caused during the forging process. This anomaly is hardly visible in the reflectance image (Fig. $4 \mathrm{~b}$ ) but is revealed by additional evaluation of polarisation information. The ridge at $u \approx 20$, running vertically in Fig. 4b, is well apparent in the reconstructed profile.

\section{Summary and Conclusion}

In this paper we have presented an image-based method for 3D surface reconstruction based on the simultaneous evaluation of reflectance and polarisation information. The reflectance and polarisation properties of the surface material have been obtained by means of a series of images acquired through a linear polarisation filter under different orientations. Analytical phenomenological models are fitted to the measurements, allowing for an integration of both reflectance and polarisation features into a unified variational framework. The method has been evaluated based on a synthetically generated surface, illustrating that for a single view and a single light source, both reflectance and polarisation features are necessary to fully recover the surface shape. We have furthermore applied the method to the $3 \mathrm{D}$ reconstruction of a raw forged iron surface, showing that the approach is suitable for detecting anomalies of the surface shape. 
The approach described in this paper relies on a single view of the surface, illuminated by a single light source. Concerning future work, we expect that a less ambiguous and more accurate reconstruction result can be obtained by taking into account multiple views and light sources, which will involve an approach based on photopolarimetric stereo.

\section{References}

1. J. Batlle, E. Mouaddib, J. Salvi. Recent progress in coded structured light as a technique to solve the correspondence problem: a survey. Pattern Recognition, vol. 31, no. 7, pp. 963-982, 1998.

2. B. K. P. Horn, M. J. Brooks. Shape from Shading. MIT Press, Cambridge, Massachusetts, 1989.

3. B. K. P. Horn. Height and Gradient from Shading. MIT technical report 1105A. http://people.csail.mit.edu/people/bkph/AIM/AIM-1105A-TEX.pdf

4. X. Jiang, H. Bunke. Dreidimensionales Computersehen. Springer-Verlag, Berlin, 1997.

5. D. Miyazaki, M. Kagesawa, K. Ikeuchi. Transparent Surface Modeling from a Pair of Polarization Images. IEEE Trans. on Pattern Analysis and Machine Intelligence, vol. 26, no. 1, pp. 73-82, 2004.

6. S. K. Nayar, K. Ikeuchi, T. Kanade. Surface Reflection: Physical and Geometrical Perspectives. IEEE Trans. on Pattern Analysis and Machine Intelligence, vol. 13, no. 7, pp. 611-634, 1991.

7. S. Rahmann, N. Canterakis. Reconstruction of Specular Surfaces using Polarization Imaging. Int. Conf. on Computer Vision and Pattern Recogntion, vol. I, pp. 149155, Kauai, USA, 2001.

8. C. Wöhler, K. Hafezi. A general framework for three-dimensional surface reconstruction by self-consistent fusion of shading and shadow features. Pattern Recognition, vol. 38, no. 7, pp. 965-983, 2005.

9. L. B. Wolff. Constraining Object Features Using a Polarization Reflectance Model. IEEE Trans. on Pattern Analysis and Machine Intelligence, vol. 13, no. 7, pp. 635657, 1991.

10. L. B. Wolff. Polarization vision: a new sensory approach to image understanding. Image and Vision Computing, vol. 15, pp. 81-93, 1997. 